\title{
Aortic stenosis is largely a boney affair
}

\author{
Friedrich C. Luft
}

Published online: 6 January 2015

(C) Springer-Verlag Berlin Heidelberg 2014

Aortic stenosis has become the commonest valvular heart disease in the industrialized world. Valve narrowing is commonly caused by age-related progressive calcification of a normal (three-leafed) aortic valve in more than $50 \%$ of cases in patients with a mean age of 65 to 80 years. Other causes of aortic stenosis include calcification of a congenital bicuspid aortic valve that accounts for $30-40 \%$ of cases and acute rheumatic fever post-inflammatory disease, accounting for less than $10 \%$ of cases. The human aortic valve normally consists of three leaflets (trileaflets) and has an orifice of 3.0$4.0 \mathrm{~cm}^{2}$. In aortic stenosis, because of calcification, the opening of the aortic valve becomes narrowed and the leaflets immobile or stenotic (Fig. 1). Degenerative aortic stenosis, the most common variety, and bicuspid aortic stenosis both begin with damage to endothelial cells from increased mechanical stress. Inflammation is believed to be involved in the earlier stages of the pathogenesis of aortic stenosis, and associated risk factors promote the deposition of low-density lipoprotein (LDL) cholesterol and lipoprotein(a) cholesterol into the aortic valve, resulting in significant damage and stenosis over time (reviewed in [1]).

Earlier calcific aortic stenosis was believed to be a passive, degenerative process, whereas now, the progression of calcification in valvular tissue is considered to be actively regulated [2]. The identification of osteoblast-like and osteoclast-like cells in human valvular tissue caused a major shift in thinking. Mouse models of calcific aortic stenosis have facilitated mechanistic studies examining the contributions of true ectopic osteogenesis, nonosseous calcification, and ectopic osteoblast-like cells. These cells appear to function differently from skeletal osteoblasts, particularly when exposed to

\section{F. C. Luft $(\bowtie)$}

Experimental and Clinical Research Center, a joint cooperation between the Max-Delbrück Center for Molecular Medicine and the Charité Medical Faculty, Berlin, Germany

e-mail: luft@charite.de valvular dysfunction. Recent studies also suggest that valvular fibrosis and concomitant calcification may play an important role in restricting cusp movement, suggesting that calcific aortic stenosis could be more appropriately viewed as a fibrocalcific disease [3]. High-resolution echocardiography and magnetic resonance imaging have emerged as useful tools for testing the efficacy of pharmacological and genetic interventions in vivo.

Several disease-associated genes have been identified in humans and mice [4]. For instance, mutations interfering with epithelial-to-mesenchymal transformation (EMT) predispose to formation of a congenitally bicuspid valve and may predispose to calcific aortic stenosis. Knockdown of activin receptor-like kinase-2 (Alk2) perturbs valve development after EMT and predisposes to the development of a functionally bicuspid aortic valve, but does not produce overt valve calcification. Normal aging is associated with mild aortic valve sclerosis. However, in the presence of valve injury, or diseaseprone genes, congenitally normal valves can develop overt calcific aortic stenosis. Valve calcification can occur via processes that resemble bone formation. However, valve calcification can also occur by nonosteogenic mechanisms, such as formation of calcific apoptotic nodules. Anticalcific interventions might preferentially target either osteogenic or nonosteogenic calcification. In addition, both innate and acquired immunity are likely to contribute to calcific aortic stenosis. Angiogenesis is not only a feature of inflammation but may also contribute independently to progression of calcific aortic stenosis, possibly by actions of pericytes that are associated with new blood vessels [4].

Biglycan belongs to the family of small leucine-rich repeat proteoglycans, which are found in a variety of extracellular matrix tissues including the bone, cartilage, and tendon. The core protein of biglycan consists of leucine-rich repeat regions. The amino terminal end of biglycans contains two glycosaminoglycan (GAG) chains consisting of either 


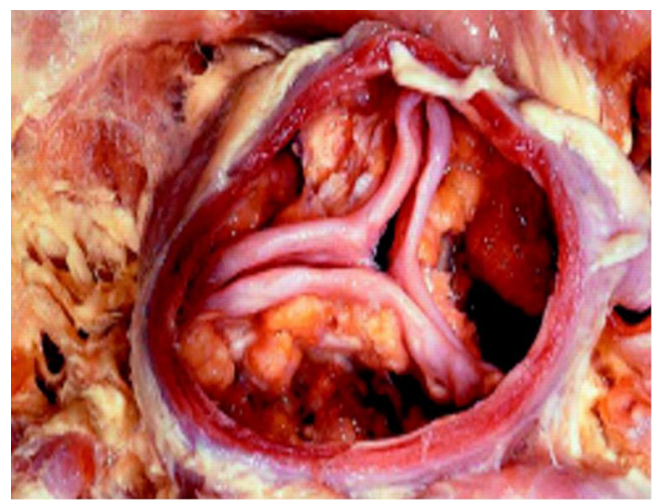

Fig. 1 Diseased aortic valve at replacement is shown. The excrescences below the valve leaflets are calcium-filled fibrous plaque-like structures resembling atherosclerotic plaques (image is from [12])

chondroitin sulfate (CS) or dermatan sulfate (DS), with DS being more abundant in most connective tissues. In human biglycan, the CS/DS chains are attached at amino acids 5 and 10. The composition of the GAG chains varies according to the tissue of origin. Nonglycanated forms of biglycan (no GAG chains) increase with age in human articular cartilage (reviewed in [5, 6]). Derbali et al. [7] conducted a geneexpression microarray study of diseased and normal aortic valves. They identified biglycan and phospholipid transfer protein (PLTP) that were overexpressed in diseased valves. Their immunohistochemical analysis revealed a colocalization of biglycan, PLTP, and Toll-like receptor-2 (TLR-2) in calcific aortic stenotic valves. In vitro, the authors showed that biglycan induced PLTP in valvular interstitial cells via the stimulation of TLR-2. Their results suggest that intricate links between valve matrix proteins, inflammation, and lipid retention are involved in the pathogenesis of calcific aortic stenosis.

In this issue of Journal of Molecular Medicine, Song et al. [8] investigated factors responsible for biglycan-induced proosteogenic reprogramming in human aortic valve interstitial cells. Biglycan induced the secretion of bone morphogenic protein-2 (BMP-2) and transforming growth factor-beta1 (TGF- $\beta 1$ ). TGF- $\beta 1$ induction was dependent upon TLR-4, rather than TLR-2. Suppression of either BMP-2 or TGF- $\beta$ reduced the expressions of alkaline phosphatase (ALP), osteopontin, and Runt-related transcription factor-2 (Runx2). When BMP-2 and TGF- $\beta$ were neutralized, osteogenic biomarkers and calcium deposition were eliminated. Biglycan administration also resulted in Smad1 and Smad3 phosphorylation. Suppressing these cytokine-receptor-signaling intermediate proteins also reduced osteogenic biomarkers and calcification. The authors conclude that concurrent upregulation of BMP-2 and TGF- $\beta 1$ is responsible for biglycan-induced pro-osteogenic reprogramming in human aortic valve interstitial cells. The Smad1/3 pathways appear to be involved in the mechanism of aortic valve interstitial cell pro-osteogenic reprogramming.

BMP-2 belongs to the TGF- $\beta$ superfamily of proteins. BMP-2, like other bone morphogenetic proteins, plays an important role in the development of bone and cartilage.
Fig. 2 A proposed model of biglycan (sine waves), BMP-2 (red triangles), and TGF- $\beta 1$ (blue diamonds) signaling in manipulating aortic valvular interstitial cells is given. Biglycan ostensibly signals via TLRs, activating NF-KB pathways Pro-osteogenic reprogramming includes BMP-2 and TGF- $\beta 1$ expression, which signal through Smads (adapted from [11])

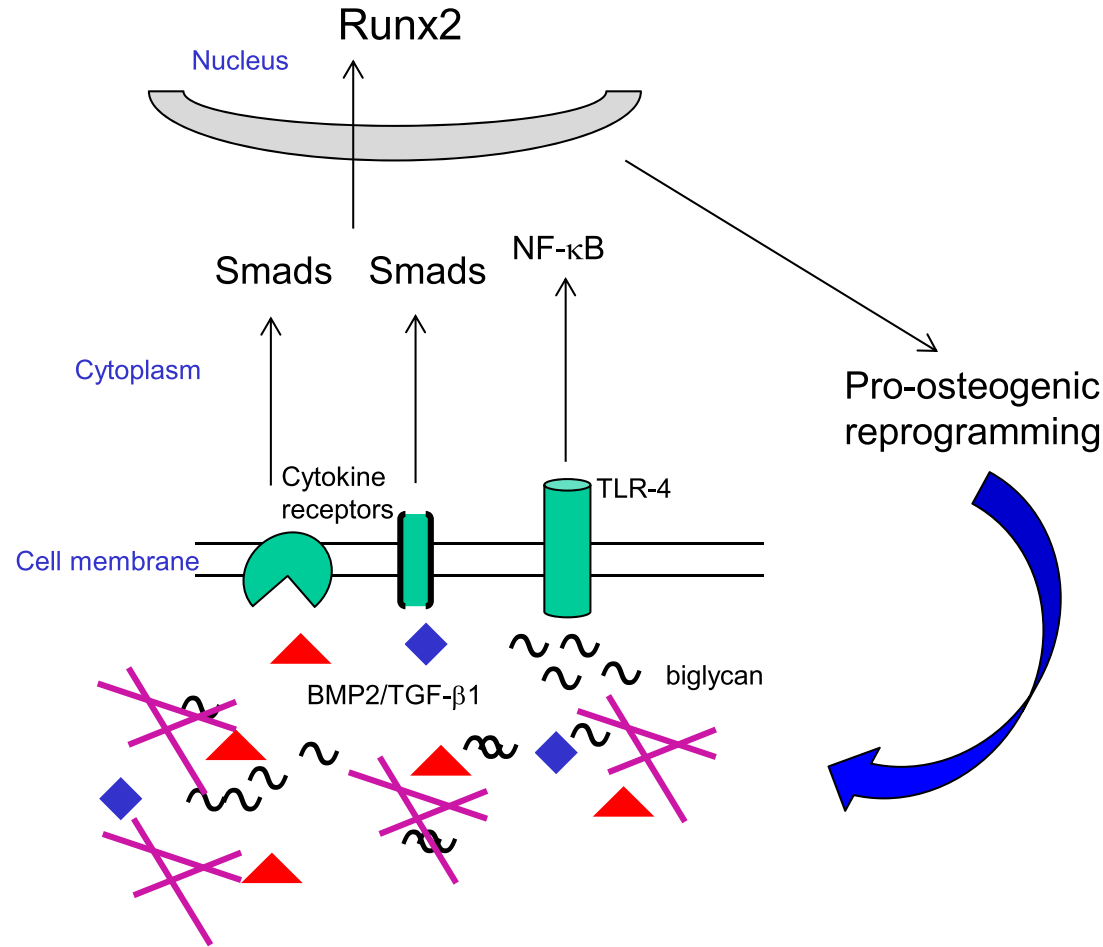


BMP-2 is involved in the hedgehog pathway, TGF- $\beta$ signaling pathway, and in cytokine-cytokine receptor interaction. The protein is involved also in cardiac cell differentiation and EMT regulation. TGF- $\beta 1$ is a polypeptide member of the transforming growth factor beta superfamily of cytokines. TGF- $\beta 1$ is a secreted protein that performs many cellular functions, including the control of cell growth, cell proliferation, cell differentiation, and apoptosis. Smads are intracellular proteins that transduce extracellular signals from TGF- $\beta$ ligands to the nucleus where they activate downstream gene transcription. The Smads are homologs of both the Drosophila proteins, mothers against decapentaplegic (MAD) and the Caenorhabditis elegans protein, small body size (SMA) (reviewed in [9]).

The current findings fit well with the authors' earlier study [10]. In the earlier paper, they reported that biglycan stimulation of cells from normal valves increased BMP-2 and ALP expression and caused accumulation of calcium deposits. TLR-2 silencing reduced BMP-2 and ALP levels after biglycan stimulation, while TLR-4 silencing did not, even though co-immunoprecipitation revealed that biglycan interacted with both TLR-2 and TLR-4. Biglycan induced the phosphorylation of extracellular signal-regulated protein kinase-1/2 (ERK1/2), p38 mitogen-activated protein kinase activation, and nuclear factor- $\mathrm{KB}$ (NF- $\mathrm{kB}$ ) expression. When ERK1/2 was inhibited, this cascade was reduced as well. Stimulating aortic valve interstitial cells with oxidized LDL cholesterol upregulated biglycan expression and release. Knockdown and neutralization of biglycan reduced the effect of oxLDL on BMP-2 and ALP expression.

Biglycan deficiency causes age-related osteopenia with a phenotype that resembles osteoporosis and premature arthritis. Biglycan is important for the differentiation of osteoblasts by increasing core-binding factor alpha-1 (CBFA-1) expression via Smad signaling [11]. This process also involves BMP-4. The finding that biglycan induces pro-osteogenic reprogramming in aortic valve interstitial cells is interesting and may improve the understanding of the mechanism of valvular calcification. A signaling schema can be formulated as shown in Fig. 2. The question remains whether or not the transformation involves an increased biglycan expression in aortic valvular tissue and how this overexpression might come about. Alternatively, other factors are upregulated sufficient to cooperate with biglycan to initiate the valvular disease process or to promote disease progression.

Respectfully,

Friedrich C. Luft

Experimental and Clinical Research Center,

A joint cooperation between the Max-Delbrück Center and the Charité Medical Faculty

\section{References}

1. Otto CM, Prendergast B (2014) Aortic-valve stenosis-from patients at risk to severe valve obstruction. N Engl J Med 371:744-756

2. Miller JD, Weiss RM, Heistad DD (2011) Calcific aortic valve stenosis: methods, models, and mechanisms. Circ Res 108(11): 1392-1412

3. Rajamannan NM et al (2011) Calcific aortic valve disease: not simply a degenerative process: a review and agenda for research from the National Heart and Lung and Blood Institute Aortic Stenosis Working Group. Executive summary: calcific aortic valve disease2011 update. Circulation 124(16):1783-1791

4. Weiss RM, Miller JD, Heistad DD (2013) Fibrocalcific aortic valve disease: opportunity to understand disease mechanisms using mouse models. Circ Res 113(2):209-222

5. Roughley PJ (2006) The structure and function of cartilage proteoglycans. Eur Cell Mater 12:92-101

6. Wilda M, Bächner D, Just W, Geerkens C, Kraus P, Vogel W, Hameister H (1998) Leucine-rich repeat glycoproteins of the extracellular matrix. Matrix Biol 17(1):1-19

7. Derbali $\mathrm{H}$ et al (2010) Increased biglycan in aortic valve stenosis leads to the overexpression of phospholipid transfer protein via Tolllike receptor 2. American J Pathol 176(6):2638-2645

8. Song R, Fullerton DA, Ao L, Zheng D, Zhao K, Meng X (2014) BMP-2 and TGF-b1 mediate biglycan-induced pro-osteogenic reprogramming in aortic valve interstitial cells. J Mol Med. doi:10. 1007/s00109-014-1229-z

9. Heldin CH, Miyazono K, ten Dijke P (1997) TGF-beta signalling from cell membrane to nucleus through SMAD proteins. Nature 390(6659):465-71

10. Song R et al (2012) Biglycan induces the expression of osteogenic factors in human aortic valve interstitial cells via Toll-like receptor-2. Arterioscler Thromb Vasc Biol 32(11):2711-2720

11. Chen XD, Fisher LW, Robey PG, Young MF (2004) The small leucine-rich proteoglycan biglycan modulates BMP-4-induced osteoblast differentiation. FASEB J 18(9):948-958

12. Image of stenotic aortic valve Queen's University 2011 [online] [viewed 2 Dec 2014] Available from: http://deptmed.queensu.ca/ $\mathrm{b} \log / \mathrm{p}=248$ 